\title{
Las políticas distributivas en Colombia: de los auxilios parlamentarios a las partidas de inversión social regional o "mermelada tóxica"
}

\author{
Distributive policies in Colombia: from parliamentary \\ aid to the social regional investment budget provisions \\ or "mermelada tóxica" (toxic marmalade)
}

Luis Alfredo Rodríguez Valero

Magister en Ciencia Política

Escuela Superior de Administración Pública, Bogotá.

Correo electrónico: Irodriguezvalero@gmail.com

\author{
Néstor Maldonado Gómez \\ Magister en Intervención Social y Derechos Humanos \\ Universidad Santo Tomás, Bogotá. \\ Correo electrónico: nestorinternacional@yahoo.es
}




\section{Resumen}

Colombia ha conocido tres modelos de políticas distributivas (PD). Cada uno de ellos dirigidos a la redistribución de recursos públicos con criterios que se debaten entre lo político y lo técnico y entre las normas formales e informales. Cada sistema ha desarrollado un diseño institucional y organizacional que promueve cierto tipo de relaciones intergubernamentales (RIG) y de relaciones entre los poderes públicos ejecutivo y legislativo. Paradójicamente, al perseguir la eliminación de las pork barrel politics, el sistema actual es el que otorga mayor discrecionalidad al ejecutivo nacional y el menos transparente. Este texto aclara algunos aspectos sobre las PD, realiza una genealogía sobre los modelos en el país y describe a profundidad cada uno en cuanto su diseño institucional, organización administrativa y montos manejados posiblemente por los legisladores, usando también la comparación con otros países. Concluye con la necesidad de emprender líneas de investigación relacionadas con el tema que aporten insumos valiosos de tipo conocimiento aplicado.

\section{Palabras clave:}

Política distributiva, relaciones intergubernamentales, estudios legislativos, descentralización, mermelada o cupos indicativos.

\section{Abstract}

Colombia has known three models of distributive policies (PD). Each one of them aimed at the redistribution of public resources based on criteria that swings between the political and the technical, and the formal and informal norms. Each system has developed an institutional and organizational design that promotes a certain type of intergovernmental relationships (RIG) and relationships between the executive and the legislative public powers. Paradoxically, in pursuit of the elimination of pork-barrel politics, the current system is the one that allows greater discretion to the executive power and is the least transparent. This text clarifies some aspects of distributive policies, makes a genealogy about the models in the country and extensively describes each one in terms of its institutional design, administrative organization and amounts possibly managed by legislators, also employing comparisons to other countries. It concludes with the need to pursue lines of research related to the subject, which provide valuable applied knowledge inputs.

Cómo citar este artículo:

Rodríguez, L. A., \& Maldonado, N. (2020). Las políticas distributivas en Colombia: de los auxilios parlamentarios a las partidas de inversión social regional o "mermelada tóxica". Revista de la Facultad de Derecho y Ciencias políticas, 50 (132), pp. 1-28. doi: http://dx.doi.org/10.18566/rfdcp. v50n132.a01

Recibido: 13 de mayo de 2019.

Aprobado: 01 de noviembre de 2019. 


\section{Key Words}

Distributive policies; intergovernmental relationships; legislative studies; decentralization; Pork barrel politics.

\section{Introducción}

Con frecuencia la ciudadanía colombiana es informada sobre posibles escándalos de corrupción por parte de los legisladores del país, al chantajear al ejecutivo nacional para el trámite de su agenda legislativa a cambio del control de asignaciones presupuestarias, mientras se interviene informal e indebidamente en la celebración de contratos de obras de inversión pública. Desde la esfera pública esta práctica ha sido denominada como: cupos indicativos o mermelada tóxica ${ }^{1}$; si bien como efecto positivo facilitan la gobernabilidad, como efectos negativos presenta el uso ineficiente, inequitativo y politizado de los recursos públicos.

Los cupos indicativos o mermelada son apenas una de las múltiples y diversas formas que pueden adoptar las políticas distributivas (PD) para la transferencia de recursos entre niveles de gobierno con base en criterios que se debaten entre lo técnico racional, lo político electoral y las normas formales e informales.

Por consiguiente, en el contexto de demandas de reformas al sistema, la presente investigación tiene por objetivo general comparar los tipos de PD que han tenido vigencia en Colombia y, como objetivos específicos describir las normas y el diseño organizacional del mismo; además de realizar estimaciones sobre los posibles montos manejados por los congresistas.

La pregunta de investigación será ¿cuáles han sido los tipos de PD que han tenido vigencia en Colombia y los efectos para las relaciones ejecutivo legislativo y para las relaciones intergubernamentales (RIG)?

1 En Colombia estos dos términos han sido usados como sinónimos al concepto de pork barrel; no obstante, ambos surgieron para describir, al menos en lo formal, fenómenos diferentes. El término de los cupos indicativos asociado al Presupuesto de Inversión Social Regional (2001) y el término de mermelada durante el debate de modificación al Sistema General de Regalías (Restrepo, 2014 b). 
Para responder estas preguntas, se presentará en primer lugar los conceptos sobre las PD, los enfoques a través de los que se ha abordado y sus principales hallazgos. Posteriormente, se revisará de manera crítica los modelos de PD que han tenido vigencia en Colombia, es decir, los Auxilios Parlamentarios (AP), el Sistema Nacional de Cofinanciación (SNC) y las Partidas de Inversión Social Regional (PISR), a fin de contrastar su diseño institucional, organización administrativa y montos manejados frente a otras experiencias internacionales donde, a diferencia del caso colombiano, se reconoce a los congresistas como actores claves y con participación formal. También se encontrarán reflexiones sobre algunos de los efectos de los modelos colombianos sobre las relaciones del poder ejecutivo- legislativo y sobre las RIG.

Se concluye con los principales hallazgos de la investigación y se deja recomendaciones sobre la necesidad de abrir líneas de investigación relacionada con las PD desde un enfoque de RIG y de la administración pública en Colombia.

La metodología de investigación utilizada es de tipo cualitativo descriptivo - estudio de caso-, con un enfoque de diseño institucional y sociología jurídica. Se utiliza principalmente una revisión de la literatura sobre las PD existente en Colombia y en el Mundo, la normatividad, sentencias, Documentos CONPES, leyes de presupuesto y los presupuestos de ISR, además de algunos archivos de fuentes periodísticas.

\section{Antecedentes, marco teórico y conceptual sobre las PD}

Desde diversas áreas de la ciencia y el saber se ha estudiado el tema de las PD, principalmente en el mundo, en mucho menor medida en Colombia. Entre ellas se destaca la ciencia política, que enfatiza la relación entre las PD, las elecciones y la representación y las ha definido con el siguiente concepto:

Aquellas que envuelven impuestos y transferencias, y en particular la decisión sobre focalización de bienes y servicios gubernamentales en localidades y grupos identificables. La manera general de pensar esto es como las autoridades públicas confieren beneficios geográficamente concentrados mientras difunden los costos a todos los votantes (Golden y Min, 2013, p. 74). 
Además de compartir el concepto de PD, vale la pena aclarar que la presente investigación se enfoca en las transferencias de recursos entre niveles de gobierno que le permiten al ejecutivo o a su coalición un cierto grado de discrecionalidad en la decisión (por ejemplo, la cofinanciación en Colombia) y no en aquellas transferencias en la que, al menos en principio, el ejecutivo nacional no tiene poder de decisión (por ejemplo, el Sistema General de Participaciones -SGP y el Sistema General de Regalías -SGR en el caso colombiano).

El texto citado de Golden y Min (2013) constituye un estado del arte del tema, donde se describen las dos principales teorías relacionadas con la democracia para explicar el fenómeno: accountability y government responsiveness. También Jiménez Patiño (2015) agrupa y clasifica las investigaciones en subcategorías.

Cabe destacar que los estudios sobre las PD desde la ciencia política tienden a centrarse en la relación entre el poder ejecutivo a nivel nacional y el poder legislativo nacional como principales decisores de las PD. No obstante, trabajos como el de González y Mamone (2015), Bertelli y Grose (2009) han comenzado a incluir nuevos actores. Así, por ejemplo, González y Mamone (2015) dan respuesta a la pregunta sobre el peso de los gobernadores y la coalición de gobierno en la recepción de asignaciones. Por su parte, el estudio de Bertelli y Grose (2009) explicaba que la asignación de earmarks² dependía de varios factores, donde el ideológico partidista es uno.

También Hána (2014) aborda los efectos de las PD en los alcaldes para buscar recursos en República Checa. Según los datos hay una respuesta generalizada frente a la importancia de contar con "buenas relaciones" con los Congresistas para la gestión de la transferencia de recursos.

Por su parte, desde la economía política Hallberg, Scartascini y Stein (2010) sostienen que para estudiar el tema sobre "El proceso presupuestario no debe analizarse como parte de una discusión técnica y aislada, por el contrario, es política” (Hallberg, Scartascini y Stein, 2010, p. 5).

Más cercano a los objetivos de la presente investigación, desde las RIG y la administración y gestión pública también ha sido abordado el tema. El trabajo de Leyva Botero (2011) describe la evolución teórica de las RIG, la importancia

2 Partidas de gasto del nivel nacional para las regiones incluidas por los Congresistas en EEUU. 
de las normas formales en la asignación de competencias y coordinación claras entre niveles de gobierno. Destaca que la ausencia de mecanismos formales de coordinación y cooperación entre los niveles de gobierno lleva a la consolidación de una "explotación oportunista" por parte de un nivel de gobierno sobre otro.

Respecto de los trabajos de administración pública, Sciara (2009) estudia la relación entre los earmark y la planificación de los entes locales y señala que existe una aparente desconexión entre ambas. Su trabajo consiste en un análisis sobre lo administrativo, lo organizacional y del funcionamiento de la labor presupuestaria del Congreso y de las oficinas locales de planeación para dar luces sobre una “caja negra”.

Finalmente, con un enfoque interdiciplinario entre las RIG y la administración pública se cuenta con el libro editado por Baskin y Mezey (2014) sobre los Constituency Development Funds (CDF), que estudia este tipo de PD en las que se permite formalmente la participación de los legisladores. Los autores analizan con un profundo nivel de detalle los modelos de PD de varios países. Concluyen que es mucho mejor contar con modelos formales y equitativos de asignación de gasto, que modelos más discrecionales, informales y menos transparentes.

Desde estos mismos campos del saber existen ejemplos de investigación que tienen por objeto de estudio el caso colombiano y que abordan de manera tangencial o principal las PD:

Así, desde la ciencia política se encuentra, en primer lugar, las investigaciones sobre el clientelismo y patrimonialismo (Leal Buitrago y Ladrón de Guevara, 1991). A través de un estudio de historia de vida describe las estrategias para el dominio de la política local y municipal, entre ellas el uso de los AP.

También desde la ciencia política cuantitativa-explicativa, Mejía, Botero y Rodríguez Raga (2008) dan respuesta a la pregunta sobre los motivos detrás de la inversión en infraestructura vial durante el gobierno de Álvaro Uribe 20022006. Entre sus hallazgos señalan que el factor principal para la asignación recursos no fue el pork barrel; sino el neo-populismo, como relación entre la asignación de proyectos y las demandas durante consejos comunitarios.

Vale la pena destacar el trabajo de Galvis, Medina y Pallares (2009) sobre la relación entre el poder de decisión de las coaliciones regionales en el 
Congreso y la aprobación del Plan Nacional de Inversiones Públicas durante las discusiones de los Planes Nacionales de Desarrollo PND cada cuatro años.

Finalmente, desde un enfoque cuantitativo- explicativo se cuenta con el texto de Bonilla Mejía e Higuera Mendieta (2017), quienes tienen por objetivos dar cuenta de la ventaja electoral producto de la afiliación política y de la recepción de transferencias a el nivel municipal entre 1997 y 2015. Los principales hallazgos se pueden resumir en que pertenecer a la coalición de gobierno nacional genera mayores transferencias por concepto de cofinanciación para los alcaldes y municipios (mientras es irrelevante estadísticamente para SGP y SGR) $)^{3}$.

Ahora bien, otro trabajo con un enfoque cualitativo es el de Valencia y Ávila (2014), quienes describen el funcionamiento de las redes políticas para la captura del Estado por medio de las elecciones en Colombia. En tanto se usa entre sus principales estrategias el clientelismo y patrimonialismo, financiadas con recursos de los PIRS.

Desde la economía política, Vargas (1999) analiza las relaciones entre el poder ejecutivo y legislativo en Colombia, el modelo de la separación de poderes y los costos para llegar a acuerdos, con especial énfasis en los AP. Frente a esto señala que la eliminación en 1991 de los AP "generales o predefinidos" abrió la puerta al aumento de los AP "personalizados o negociados", menos transparentes y con más efectos negativos ${ }^{4}$. Paralelamente, desde la economía política, Pubiano y López (2004), Cárdenas y Mejía y Olivera (2006) responden al cuestionamiento sobre la modernidad del modelo presupuestario colombiano. En esta misma línea y enfocándose en la evaluación del SNC se cuenta con las investigaciones de Corzo (1997), Leguizamo (1997) y de González (2001). Cabe destacar que los tres trabajos tienen un sesgo técnico y antipolítico que puede desvirtuar el diagnóstico y la propuesta de soluciones reales a un tema político. Una postura contraria se puede encontrar en el texto de Gómez Barón (2003).

\footnotetext{
3 Los autores señalan que esto último puede responder a que la cofinanciación apenas representa $3.2 \%$ del total de inversiones en los municipios y apenas $10 \%$ de la inversión sobre vías y carreteras (variable dependiente del estudio).

4 Los AP "generales o predefinidos" serán los descritos en estas líneas en el apartado 2.1, mientras que los AP "personalizados o negociados" está descritos en las presentes líneas en los apartados 2.2 y 2.3 .
} 
Finalmente, desde el derecho existen muy pocos estudios directamente relacionados con las PD. Estos se enfocan en el tema del trámite formal del presupuesto (Restrepo, 2014 a; Ramírez Plazas, 2015; Sánchez Cubides, 2015; Ortega Cárdenas, 2010). No obstante, existen algunos trabajos que se han encargado de analizar el tema desde una perspectiva socio- jurídica como son Aponte Olivella (1983), Durán y Montenegro (2002).

En consecuencia, queda claro que la mayoría de la literatura ha sido producto de estudios de la ciencia política y en menor medida de la economía política; mientras que desde otros campos como la RIG, la administración pública y el derecho, el estudio es apenas incipiente, al menos para el caso colombiano.

\section{De los auxilios parlamentarios a las partidas de inversión social regional: Descripción, precisiones y comparación con otros modelos de PD del mundo}

Es importante señalar que, tanto la Constitución Política de Colombia de 1886 como la Constitución Política de 1991 de Colombia reconocen como forma de Estado una República unitaria. Por tanto, de entrada, se observa que las autonomías legislativa y fiscal de los entes del nivel territorial estarán limitadas.

Lo anterior no es una tendencia única del caso colombiano, de hecho, tanto en la región (Ugalde, 2011), como en países con democracias federales con alto nivel de ingreso (Argullol Murgadas, 2004), el fenómeno se ha acentuado con la diferencia que en estos países se hace bajo un marco de RIG de coordinación.

En cuanto a las relaciones interfiscales, es decir, las formas en las que el nivel nacional transfiere recursos a los niveles inferiores de gobierno, la Constitución Política de Colombia de 1991 señala el SGP (Art. 357), el SGR (Art. 360) y la cofinanciación (Art. 351).

\subsection{Los auxilios parlamentarios (1886-1991)}

A partir de lo anterior, se comienza con la descripción de la política distributiva de los AP. Debe señalarse que estuvieron vigentes durante el 
período en que rigió la Constitución Política de Colombia de 1886 a 1991. Su existencia encontraba sustento en artículos de rango constitucional sobre las funciones del Congreso de la República, específicamente el artículo 78 numeral 20: "Art. 78 numeral 20: Fomentar las empresas útiles o benéficas dignas de estímulo o apoyo" (Constitución Política de Colombia de 1886)

Esta facultad y atribución fue reconocida también para los diputados de las Asambleas Departamentales y concejales del Distrito Especial de Bogotá en el artículo 185 de la Constitución Política de 1886 (Constitución Política, 1886, art. 185) y sus reformas.

Procedimentalmente, para ajustarse a la normatividad que regula la hacienda y el gasto público, los AP se reflejaban en los Decretos de Liquidación de los presupuestos anuales bajo el nombre de auxilios o en la sección de transferencias (Durán y Montenegro, 2002).

Los recursos eran recibidos por la tesorería municipal y esta se encargaba de entregarlo a las personas públicas y privadas que indicaran congresistas, diputados y concejales. A su vez, estos receptores debían entregar informes sobre la utilización de los recursos ante los órganos de control.

Ahora bien, conforme la normatividad del sistema, su diseño y las razones que explican el mismo se analizará los valores manejos y sus los efectos del sistema. A pesar de la dificultad de manejar estos datos históricos en la literatura, Leal y Dávila (1991) dejan una pequeña referencia: 
Gráfica 1. Ingresos del Municipio Rionegro, Santander de 1960 a 1986

\begin{tabular}{|c|c|c|c|c|c|c|c|}
\hline \multicolumn{8}{|c|}{$\begin{array}{c}\text { Cuadro } 14 \\
\text { Ingresos del municipio de Rionegro por Tesorería. } \\
\text { 1960-1986 (pesos de } 1975 \text { y porcentajes) (1) }\end{array}$} \\
\hline Año & $\begin{array}{l}\text { Impuesto } \\
\text { Predial } \\
\quad(2)\end{array}$ & $\begin{array}{c}\text { Industria } \\
\mathbf{y} \\
\text { Comercio } \\
\text { (3) }\end{array}$ & $\begin{array}{l}\text { Participación } \\
\text { impuesto a } \\
\text { las ventas (4) }\end{array}$ & $\begin{array}{l}\text { Por } \\
\text { explotación } \\
\text { petrolera } \\
\text { (5) }\end{array}$ & $\begin{array}{c}\text { Otros } \\
\text { ingresos } \\
\quad(6)\end{array}$ & $\begin{array}{l}\text { Auxilios } \\
\text { Departa } \\
\text { mentales y } \\
\text { Nacionales (7) }\end{array}$ & Total \\
\hline \multirow{2}{*}{$\begin{array}{c}1960 \\
(8)\end{array}$} & 937,544 & 88,612 & 0 & 0 & 628,578 & 204,082 & $1,858,816$ \\
\hline & 50 & 4.8 & 0 & 0 & 33.8 & 11 & 100 \\
\hline \multirow{2}{*}{$\begin{array}{c}1964 \\
(8)\end{array}$} & 764,275 & 88,443 & 0 & 118,127 & 542,480 & 534,176 & $2,047,501$ \\
\hline & 37.3 & 4.3 & 0 & 5.8 & 26.5 & 26.1 & 100 \\
\hline \multirow{2}{*}{$\begin{array}{c}1968 \\
(8)\end{array}$} & $1,302,439$ & 100,762 & 0 & $1,108,304$ & $1,016,550$ & 635,359 & $4,163,414$ \\
\hline & 31.3 & 2.4 & 0 & 26.6 & 24.4 & 15.3 & 100 \\
\hline \multirow[t]{2}{*}{1970} & $1,272,465$ & 107,021 & 0 & $2,034,067$ & $1,068,782$ & $1,104,167$ & $5,586,502$ \\
\hline & 22.8 & 1.9 & 0 & 36.4 & 19.1 & 19.8 & 100 \\
\hline \multirow[t]{2}{*}{1971} & $1,420,027$ & 78,272 & 121,144 & $2,192,019$ & 984,184 & $1,014,644$ & $5,810,290$ \\
\hline & 24.4 & 1.3 & 2.1 & 37.7 & 16.9 & 17.5 & 100 \\
\hline \multirow[t]{2}{*}{1972} & $1,710,956$ & 63,326 & 0 & $1,752,170$ & 786,913 & 808,750 & $5,122,115$ \\
\hline & 33.4 & 1.2 & 0 & 34.2 & 15.4 & 15.8 & 100 \\
\hline \multirow[t]{2}{*}{1973} & $1,155,008$ & 111,672 & 956,085 & $1,346,162$ & $1,110,066$ & 569,507 & $5,248,500$ \\
\hline & 22 & 2.1 & 18.2 & 25.6 & 21.2 & 10.9 & 100 \\
\hline \multirow[t]{2}{*}{1974} & $1,100,821$ & 106,224 & $1,609,867$ & $1,176,538$ & $1,219,311$ & 849,294 & $6,062,055$ \\
\hline & 18.2 & 1.8 & 26.6 & 19.4 & 20.1 & 14 & 100 \\
\hline \multirow[t]{2}{*}{1975} & 911,912 & 93,137 & $2,382,008$ & $1,460,436$ & $1,061,769$ & $1,576,000$ & $7,485,262$ \\
\hline & 12.2 & 1.2 & 31.8 & 19.5 & 14.2 & 21.1 & 100 \\
\hline \multirow[t]{2}{*}{1976} & 420,002 & 51,108 & $2,420,831$ & 795,394 & 472,934 & 0 & $4,160,269$ \\
\hline & 10.1 & 1.2 & 58.2 & 19.1 & 11.4 & 0 & 100 \\
\hline \multirow[t]{2}{*}{1977} & 913,687 & 87,788 & $1,651,991$ & 519,081 & $1,357,906$ & 0 & $4,530,453$ \\
\hline & 20.2 & 1.9 & 36.5 & 11.5 & 30 & 0 & 100 \\
\hline \multirow[t]{2}{*}{1978} & $1,147,773$ & 151,903 & $1,733,041$ & 465,978 & $1,324,908$ & 0 & $4,823,603$ \\
\hline & 23.8 & 3.1 & 35.9 & 9.7 & 27.5 & 0 & 100 \\
\hline \multirow[t]{2}{*}{1979} & $1,589,419$ & 137,266 & $1,520,600$ & 277,314 & 817,382 & 0 & $4,341,981$ \\
\hline & 36.6 & 3.2 & 35 & 6.4 & 18.8 & 0 & 100 \\
\hline \multirow[t]{2}{*}{1980} & $2,402,451$ & 123,170 & $2,564,806$ & 109,614 & $1,393,265$ & 0 & $6,593,306$ \\
\hline & 36.4 & 1.9 & 38.9 & 1.7 & 21.1 & 0 & 100 \\
\hline
\end{tabular}




\begin{tabular}{|c|c|c|c|c|c|c|c|}
\hline \multicolumn{7}{|c|}{$\begin{array}{c}\text { Cuadro 14 } \\
\text { Ingresos del municipio de Rionegro por Tesorería. } \\
\text { 1960-1986 (pesos de 1975 y porcentajes) (1) }\end{array}$} \\
\hline Año & $\begin{array}{c}\text { Impuesto } \\
\text { Predial } \\
(\mathbf{2})\end{array}$ & $\begin{array}{c}\text { Industria } \\
\mathbf{y} \\
\text { Comercio } \\
\mathbf{( 3 )}\end{array}$ & $\begin{array}{c}\text { Participación } \\
\text { impuesto a } \\
\text { las ventas (4) }\end{array}$ & $\begin{array}{c}\text { Por } \\
\text { explotación } \\
\text { petrolera } \\
\mathbf{( 5 )}\end{array}$ & $\begin{array}{c}\text { Otros } \\
\text { ingresos } \\
(\mathbf{6})\end{array}$ & $\begin{array}{c}\text { Auxilios } \\
\text { Departa } \\
\text { mentales y } \\
\text { Nacionales (7) }\end{array}$ & Total \\
\hline 1981 & $2,859,481$ & 83,392 & $4,525,347$ & 356,778 & $2,020,117$ & 0 & $9,845,115$ \\
\hline & 29 & 0.8 & 46 & 3.6 & 20.5 & 0 & 100 \\
\hline 1982 & $2,025,688$ & 168,854 & $3,441,877$ & 552,820 & $1,981,050$ & 0 & $8,170,289$ \\
\hline & 24.8 & 2.1 & 42.1 & 6.8 & 24.2 & 0 & 100 \\
\hline 1983 & $2,544,874$ & 146,749 & $2,888,482$ & $1,048,527$ & $1,901,237$ & 463,983 & $8,993,852$ \\
\hline & 28.3 & 1.6 & 32.1 & 11.7 & 21.1 & 5.2 & 100 \\
\hline 1984 & $2,963,476$ & 182,771 & $3,512,040$ & $1,536,300$ & $2,683,607$ & 753,546 & $11,631,740$ \\
\hline & 25.5 & 1.6 & 30.2 & 13.2 & 23.1 & 6.5 & 100 \\
\hline 1985 & $1,838,353$ & 197,829 & $3,087,111$ & $1,473,025$ & $3,719,354$ & $1,286,251$ & $11,601,923$ \\
\hline & 15.8 & 1.7 & 26.6 & 12.7 & 32.1 & 11.1 & 100 \\
\hline 1986 & $1,336,872$ & 151,019 & $4,581,760$ & 702,887 & $3,709,204$ & 190,846 & $10,672,588$ \\
\hline & 12.5 & 1.4 & 42.9 & 6.6 & 34.8 & 1.8 & 100 \\
\hline
\end{tabular}

Fuente:

Balance Mensual de Ingresos por Tesorería al Municipio de Rionegro 1960-1986.

NOTA:

1) Deflactado según precios implícitos del PIB.

2) Ingreso tributario cobrado por Tesorería. Depende del avalúo Catastral.

3) Ingreso tributario cobrado por la Tesorería a establecimientos industriales y comerciales del municipio.

4) Ingreso tributario transferido por el Estado central.

5) Ingreso tributario por concepto de las explotaciones petroleras que se realizan en el departamento de Santander. El traspaso de dólares a pesos se hizo para cada año con base en las tasas de cambio promedio establecidas por el Banco de la República.

6) Incluye varias clases de ingresos. Ingresos tributarios menores como avisos, almacén y sellos, degüello de ganado, ventas ambulantes y de pólvora. Ingresos no tributarios como servicios de alumbrado público, aseo y recolección de basuras. Ingresos por uso de la plaza de ferias y rentas ocasionales. La contribución de tránsito o caminos para el mantenimiento de carreteras y caminos, rentas compensadas como los cobros por acueducto, del fondo de valorización, del fondo de previsión social y de los depósitos especiales.

7) Ingreso por auxilios departamentales y nacionales conseguidos por diputados y representantes. Son cobrados por juntas, colegios, etc. En la Tesorería.

8) Estos años se tomaron espaciadamente para establecer la tendencia durante el Frente Nacional.

Fuente: Leal y Dávila (1991, p. 337 y 338).

Como puede observarse, de 1960 a 1986 los recursos de los AP y asamblearios en conjunto variaron de un mínimo de $10.9 \%$ a un máximo de $26.1 \%$, frente a la suma total de ingresos del municipio. Esto permite 
identificar que los AP fueron una de las principales fuentes de ingresos para este municipio.

Vale la pena aclarar que existe una serie de años en los cuales los auxilios departamentales y nacionales son iguales a 0 pesos (1976-1982). Al revisar el texto de Leal y Dávila (1991) la posible explicación se encuentra en el conflicto entre el "liberalismo oficial" de políticos tradicionales que controlaban a Congresistas del departamento, gobernador y, en alguna medida, a los alcaldes frente al político clientelista local quien controlaba el concejo municipal.

Finalmente, frente a los montos, para hacer una comparación con PD en otros países, se toma como referencia el año que más AP se recibieron: 1975 , fue de 1,576,000 pesos o 49,250 dólares ${ }^{5}$.

En el caso de los auxilios para concejales de Bogotá, el archivo de las actas de esta corporación permite calcular que, para el año 1982, cada concejal contaba con un cupo de 6 millones de pesos o 92,000 dólares (tasa cambiaria de ese año).

Ambas cifras colombianas parecen elevadas si se compara con la política distributiva de los Fondo para el Desarrollo de las Circunscripciones municipales de la India (CDF), que surgieron a mediados de los años 70`s en grandes ciudades como Bombay, donde se manejaba un monto de 50,000 rupias o 6,297 dólares para cada concejal por año (Blair, 2014, p. 169).

La legislación local de la India permitía que varios concejales unieran sus recursos para emprender obras de mayor envergadura (Blair 2014). Para 2011, los montos manejados por los CDF de tipo parlamentario en la India son de 417,000 dólares (Blair, 2014, p. 169). También como punto de comparación puede señalarse el caso de Filipinas de 1982, donde los Congresistas contaban en la Ley General de Apropiaciones con la partida denominada "Apoyo a Proyectos de Desarrollo" que asignaba 500,000 pesos filipinos o 54,000 dólares a cada legislador (Corte Suprema de Filipinas, 2013).

Lamentablemente, por la propia estructura de la PD colombiana, la información no siempre estaba discriminada para saber el número exacto de congresistas y diputados de la Asamblea Departamental que participaron en la gestión de dichos recursos.

5 Tasa cambiaria de ese año. 
El número de auxilios manejados por congresistas, diputados y concejales de Bogotá era variable, pues los representantes podían decidir repartir montos elevados entre pocas organizaciones o por el contrario montos pequeños entre muchas organizaciones. Según Aponte Olivella (1983), el número de AP del presupuesto del año 1983 fue de 10,207. Lo que dividido entre los 313 legisladores para la fecha da un promedio de 33 auxilios por Congresista. En el caso de Bogotá, de una muestra de 8 concejales para el presupuesto de 1982 los auxilios variaron de un mínimo de 18 (concejal Carlos Alberto Saavedra) y un máximo de 99 (concejal Enrique Silva Valdivieso).

Los receptores de los auxilios se concentraban principalmente en instituciones educativas, juntas de acción comunal y organizaciones sociales diversas, que van desde instituciones de salud hasta instituciones que protegían el patrimonio cultural, entre otras. Entre las críticas a los AP, Aponte Olivella (1983) destaca el poco control al uso de los recursos hasta antes de la Ley 20 de 1975 y problemas en el diseño: como la entrega de recursos a Juntas de Acción Comunal con poca capacidad para la gestión de proyectos y de recursos, la poca transparencia en la selección de los beneficiados de los AP por concepto de "becas" en las instituciones educativas y, finalmente, la posibilidad que los propios congresistas, diputados y concejales de Bogotá fueran beneficiarios de los auxilios al hacer parte de las juntas directivas 0 miembros de las organizaciones receptoras de recursos.

A esto se suma, las críticas estructurales que señala Aponte Olivella (1983) frente a que los recursos no promovían la equidad y se concentraban principalmente en Bogotá y en los departamentos con poblaciones amplias. Se señala, además, que en el caso de los AP de los congresistas no otorgaban igualdad de montos entre sus miembros, algo diferente al funcionamiento de los AP para concejo del distrito de Bogotá, donde todos los concejales contaban con los mismos montos y valores.

Por su parte Vargas (1999) y Gómez Barón (2003) afirman que, contra la equivocada creencia popular, se trataba de montos modestos que tenían las ventajas de ser transparentes y permitir el seguimiento y vigilancia de la ciudadanía. Una vez eliminados es claro que los costos de transacción entre ejecutivo y legislativo se elevaron mucho más, en la práctica informal y poco transparente de los Fondos para el Desarrollo Regional que, por ejemplo, para 1997 aumentó en 8 veces más los recursos manejados en 1990 (Vargas, 1999). 
Gómez Barón (2003) también critica que los AP no tuviesen mecanismos institucionales para vigilar la gestión de los recursos, aunque recuerda que para la fecha en que tuvieron vigencia los AP, en realidad no había planeación del Estado y la mayoría de sus fondos trabajaban de manera "inercial" (Gómez Barón, 2003), recuerda que el primer Plan Nacional de Desarrollo apenas se dio luego de la Constitución de 1991.

En todo caso, el listado de críticas contra los AP permitió que el discurso tecnocrático se impusiera sobre la idea de la representación y las relaciones formales entre ejecutivo y legislativo .

\subsection{El Sistema Nacional de Cofinanciación SNC (1992-1998)}

A partir de 1991, las PD cambian, en primer lugar, hacia el SNC y, posteriormente, a las partidas del PIRS, conocidas hoy como cupos indicativos o mermelada.

Y es que el artículo 355 (Constitución Política de Colombia de 1991) prohibió los AP y, a su vez, abrió la posibilidad de los convenios de interés público que constituyó un amplio listado de excepciones a la prohibición (Durán y Montenegro, 2003) .

Frente al SNC se trata de transferencias condicionadas y discrecionales (no automáticas) del ente nacional a los entes territoriales. La cofinanciación estuvo regulada por los Decreto 77-1987 y 2132-1992, recibió desarrollo jurisdiccional en la sentencia $\mathrm{C}-562-1998$. Al igual que otras PD, el sistema fue objeto de demandas de constitucionalidad. En la demanda contra el SNC el actor solicitaba la inconstitucionalidad de varios artículos a la Ley de Presupuesto 413-1997, entre ellos el artículo 55. El actor de la demanda se opuso al establecimiento de cuotas de participación a los entes y a la usurpación de la voluntad de las comunidades receptoras de la inversión.

Como respuesta a estos argumentos, la Corte Constitucional declaró inexequible solamente la sección del Parágrafo del artículo demandando sobre los porcentajes de cofinanciación del ente territorial, sin detenerse a estudiar o a emitir consideración alguna sobre los argumentos principales del actor.

Ahora bien, en cuanto a temas procedimentales, las partidas de gasto del SNC estaban constituidas de dos formas: 1) Partidas globales en el presupuesto, 
que posteriormente serán delimitadas algo más (sin garantizar la ejecución) en el Decreto de Liquidación del Presupuesto y para los que los entes territoriales debían participar y competir para obtener los recursos del SNC, de manera más o menos transparente. 2) Partidas de Asignación Específica, en que ciertos proyectos eran incluidos en el Decreto de Liquidación para obligar a que su financiación fuera con los recursos del SNC, a pesar de que estos proyectos no cumplían con el trámite formal de competencia que establecía el sistema.

Según Leguizamo (1997), este segundo mecanismo fue el usado por el ejecutivo para cumplir con acuerdos y compromisos informales con los congresistas. Según la autora, los montos de asignación específico fueron para 1995 el 35\% de todo el sistema (Leguizamo, 1997). Los fondos que habrían destinado más recursos a asignación específica dicho año fueron el FIU con 78\% de sus 84 mil millones de pesos y el FCV con 55\% de los 68 mil millones. Cabe destacar que estos dos fondos son los que contaban con la menor cantidad de recursos totales en comparación con el FIS y el DRI, el primero con 228 mil millones y el segundo con 152 mil millones (Leguizamo, 1997).

Lo anterior se diferencia bastante de los sistemas de PD que reconocen la participación de los legisladores. Por ejemplo, los Earmark en EEUU son debatidos y aprobadas en el legislativo con la especificación de los proyectos.

En otros casos, sistemas de PD más formales garantizan mayor seguridad jurídica a los legisladores y a la población que ellos representan, dado que por normas superiores tienen garantizados asignaciones de montos de dinero fijos. Por ejemplo, en Kenya los "Fondos para el Desarrollo de las Circunscripciones" están protegidos por Ley (2003), sus montos y valores están atados al ingreso anual de los impuestos (Ndii, 2014).

Al volver al diseño institucional y la estructura administrativa encargada de poner en funcionamiento el sistema, el mismo adolecía de competencias claras entre sus entes y una desconexión entre los objetivos y valores a los que aspiraba y las herramientas para hacerlo realidad. Así, el Decreto 2132-1992 señalaba la necesidad de crear varias instancias como: los Comités Nacional, Departamentales, Distritales y las $\mathrm{UDECO}^{6}$.

De entrada, se observa la poca participación comunitaria en la identificación, priorización y decisión de los proyectos a pesar que el artículo 24 numeral 9 del

6 Unidades Especializadas Departamental o Distrital de Cofinanciación. 
Decreto 2132-1992 otorgó la función de promover la participación comunitaria de estas funciones en los municipios.

El artículo 26 del Decreto 2132-1992 también obligó a la revisión y al establecimiento de normas y lineamientos de funcionamiento del sistema a los Documentos CONPES. Con base en dicha obligación se expidieron los Conpes 029-1995, 2791-1995 y 2865-1996. Estos Conpes, además de establecer lineamientos como los montos máximos a invertir por sector según los cupos indicativos de cada ente territorial, identificó un listado de problemas en el funcionamiento del SNC. Por ejemplo, el Conpes 029-1995 señaló problemas como: asignar la competencia de la participación comunitaria a los municipios sin que tuvieran capacidad; falta de acompañamiento a los órganos que conformaban el SNC en el nivel regional; problemas de coordinación entre la UDECO y los Bancos de Proyectos de Inversión (BPIN).

Por su parte el Conpes 2791-1995 señaló problemas como: falta de capacidad institucional en el nivel local para formular los proyectos; declaración de invalidez de los Convenios Interadministrativos suscritos entre los fondos y los entes territoriales; ausencia de reglamentación al funcionamiento de entes del SNC; falta de acompañamiento de los Ministerios a los entes territoriales para estudios de pre-inversión; el sistema cerrado imposibilitaba a municipios con capacidad de formulación de proyectos a participar directamente sin los UDECO $^{7}$.

Frente a los montos del SNC, éstos fueron de 534,052,000,000 pesos para el año 1995 (Conpes 2865 de 1996). Lo que correspondía al 3\% del total del presupuesto nacional para ese año de 17 billones de pesos. Los montos manejados eran considerables si se compara con las PD tipos CDF en otros países, que manejan montos de un máximo 5.48\% del total del presupuesto en Papúa Nueva Guinea (Baskin, 2014). Vale la pena destacar también que, en el caso del SNC colombiano, permitió que no sólo los entes territoriales participaran en la obtención de dichos recursos; sino la nación. Según los datos del Conpes 2865-1996 (Conpes 2865 de 1996), la nación recibió 70,322,000,000 millones de pesos. Lo que equivale a un 13\% del total de los recursos manejados por el SNC.

7 Vale la pena señalar que el Conpes 2865-1996 reconoce que buena parte de los problemas de funcionamientos del sistema fueron atendidos en coordinación entre los fondos de cofinanciación, el DNP y la Red de Solidaridad Social (Conpes 2865-1996, p. 11). 
Si se tomara el monto total del SNC entre el total de legisladores para el año 1995, es decir, 265 legisladores, los montos por legislador serían de 2,015,290,566 pesos o 2,209,748 dólares por congresista (monto muy superior a los manejados por congresistas en sistemas CDF). Si se analizara los datos de los proyectos de asignación específica de los fondos FIU y FCV, los montos por congresistas serían de 425,386 dólares (montos semejantes a los manejados por congresistas en sistemas $\mathrm{CDF}$ ).

En los casos de países con CDF, los montos promedios son:

Gráfica 2. Montos de inversión manejadas por cada legislador en países con el sistema de los CDF.

Table 1.1 Amounts Allocated per MP in USD

\begin{tabular}{lc}
\hline & $\begin{array}{c}\text { Average Amount Allocated } \\
\text { per MP (USD) }\end{array}$ \\
\hline Philippines & $\$ 4,270,001$ \\
Bhutan & $\$ 43,000$ \\
Solomon Islands & $\$ 140,000$ \\
Kenya & $\$ 794,464$ \\
Malaysia & $\$ 577,951$ \\
Jamaica & $\$ 456,361$ \\
India & $\$ 420,790$ \\
Sudan & $\$ 317,543$ \\
Pakistan & $\$ 240,000$ \\
Malawi & $\$ 21,352$ \\
Tanzania & $\$ 13,761$ \\
Uganda & $\$ 5,187$ \\
\hline
\end{tabular}

Source: Tsangana, Alison Hickey. "Constituency Development Funds Scoping Paper," International Budget Project, 18 January $2010 \mathrm{http} / / /$ internationalbudget.org/wp.content upload/Constituency-Develpoment-Funds-Scoping-Paper.pdf., pp. 4-5. The paper does not indicate for which year the allocation took place.

Fuente: Tsangana (citado en Baskin, 2014, p. 9).

Al revisar el número de proyectos presentados en el SNC, por ejemplo, para 1996 el total de proyectos fue de 14,814. Si se procediera a atribuir la totalidad de proyectos a los congresistas, se obtendría un promedio de 56 proyectos por congresista, un número elevado y que significaría un desgaste en recursos para los congresistas. Este número contrasta con los límites de número de

8 Si bien hacer esta comparación no refleja del todo la realidad por tratarse de sistemas diferentes, el CDF reconociendo la participación de legisladores y siendo transparente; mientras el SNC se caracterizaba; por lo contrario, si resulta ilustrativo y útil fijarse en como al filtrar la información sobre los montos de los proyectos SNC de asignación específica se obtiene algunos valores semejantes en ambos sistemas. 
proyectos que pueden manejar los parlamentarios en países con CDF. Por ejemplo, en Kenya anualmente cada legislador puede presentar un mínimo de 5 y un máximo de 25 proyectos con un acompañamiento de toda una serie de entidades, órganos administrativos y de la propia comunidad (Ndii, 2014).

Aunque los cálculos y la comparación parecen darle la razón a la afirmación de Durán y Montenegro (2002) en tanto los proyectos presentados al SNC, vía participación comunitaria, se veían anulados por los proyectos de iniciativa legislativa. Este no parece ser el caso, pues no guarda correspondencia lógica que municipios con alta capacidad de gestión en la presentación de proyectos requiriesen de las gestiones de los legisladores para participar de manera efectiva en el sistema.

En suma, con todas las críticas que se le pueden atribuir a la política distributiva del SNC en Colombia, esta experiencia constituye un intento de constituir RIG modernas y de respeto a la descentralización. Lamentablemente, por la tradición formalista colombiana y por un supuesto respeto al equilibrio de poderes, la participación de los legisladores fue excluida del diseño institucional y de la organización administrativa, sin que esto fuera obstáculo para la participación informal y poco transparente de aquellos. Esto es contrario a los sistemas con PD de tipo CDF, pues además de contar con una estructura administrativa que viabilice los proyectos de inversión, promover las relaciones entre los niveles de gobierno y la participación comunitaria, reconocen a los parlamentarios como un actor clave del proceso, es por esto que son incluidos en un proceso transparente, que permita la rendición de cuentas.

Obviamente entre Colombia y los países con las figuras de CDF existen muchas diferencias que dificultaría la inclusión formal de los legisladores colombianos en las PD. Podría argumentarse desde el diseño institucional electoral $^{9}$, por ejemplo, que los países con CDF están regidos por un sistema

9 Sólo se toma el sistema electoral, dado que es el único elemento en el que se observa una generalidad entre los casos de CDF, al ser el sistema electoral mayoritario para elegir al menos una de las cámaras del legislativo. En otros elementos como forma de Estado (federal o unitaria) no se encuentra generalidad, pues mientras países como Estados Unidos, India, Malaysia y Pakistán son federales, el resto son repúblicas unitarias. Tampoco existe una generalidad frente a la facultad del legislativo para enmendar presupuestos o lo que se conoce como iniciativa de gasto, pues mientras en Estados Unidos y Malawi la facultad del legislativo es plena, en Kenya, India, Pakistán y Tanzania es limitada y en el caso de Filipinas y Uganda es muy limitada al igual que el caso colombiano (2017 IBP). Tampoco se pueden encontrar generalidades en elementos como continente, herencia cultural, nivel de ingresos o población. 
electoral tipo mayoritario para la elección del legislativo, con circunscripciones pequeñas y mayor contacto entre representantes y representados. Sin embargo, en el caso colombiano cada vez se observa más, aún en el propio Senado, cómo representantes de los intereses nacionales son elegidos concentrando sus votos en unos departamentos y en muchos casos en apenas unos pocos municipios específicos.

\subsection{Los Presupuestos de Inversión Social Regional (PISR)}

Por diversos motivos, en especial por los escándalos con el SNC y la crisis fiscal de finales de los años 90 `s, el SNC fue dejado de lado, los entes que lo conformaban dejaron de existir o fueron fusionados en otras entidades con otra naturaleza y fue remplazado por la política distributiva del PISR.

El origen del PISR se dio en el año 2000, durante la discusión del proyecto de ley de presupuesto para el año 2001 (Proyecto de Ley 13/00 Cámara y 54/00 Senado) como propuesta del ex Ministro de Hacienda, Juan Manuel Santos, para negociar con los Congresistas la aprobación del presupuesto y de otros proyectos de ley importantes, entre ellos la reforma tributaria (Durán y Montenegro, 2002). Según Durán y Montenegro (2002) la medida consistía en adicionar 300,000 millones de pesos a las PISR globales para que los Congresistas gestionaran y decidieran la utilización. Conforme Durán y Montengro (2002) el ex Ministro de Hacienda Juan Manuel Santos explicó que las principales características del sistema sería la transparencia y la participación equitativa de todos los congresistas.

Entre los argumentos jurídicos utilizados para defender el nuevo sistema se encontraba: la iniciativa de gasto de los Congresistas; que la prohibición de los AP estaba circunscrito a las entidades privadas y la función de los Congresistas de gestionar recursos para las regiones según el artículo 283 Ley 5-1992 Reglamento del Congreso.

Un argumento jurídico nuevo, que presuntamente blindaba al sistema de los PISR, fue el artículo 68 del Decreto 111-1996 Estatuto Orgánico de Presupuesto, pues obligaba a que los proyectos no podían ser aprobados y ejecutados hasta que no fueran evaluados por los órganos competentes y por el BPIN.

Para el año 2001, los 300 mil millones de pesos habrían sido incluidos en el presupuesto en partidas globales con objetivos de infraestructura o construcción 
en diversos órganos de la administración nacional como Ministerios o entes adscritos o vinculados al nivel nacional ${ }^{10}$ (Durán y Montenegro, 2002).

Ahora bien, frente al funcionamiento formal del sistema (algo muy escueto y sin regulación adicional salvo las propias leyes del presupuesto de rentas), Durán y Montenegro (2002) señalan que la iniciativa de presentar proyectos estaba abierta a funcionarios de elección popular y a la propia ciudadanía. Posteriormente, se requería que el alcalde o gobernador iniciara un acta de convenio con la "entidad ejecutora" $11 \mathrm{y}$, finalmente, se firma un convenio interadministrativo para el traslado de los recursos a la entidad territorial y que ésta realice el contrato y el seguimiento del mismo.

Por su parte, el funcionamiento informal, según investigaciones realizadas por La Silla Vacía (2018), comenzaría con la negociación entre enlaces de los Ministerios con cada congresista de las partidas de inversión a cambio de apoyo en iniciativas legislativas. Posteriormente, los congresistas ordenan a alcaldes o gobernadores aliados que presenten proyectos de inversión en esas entidades. Finalmente, el enlace del ministerio presiona a la entidad para la aprobación del proyecto y la transferencia de recursos. De acá en adelante se adelanta los procesos de contratación.

Como se observa, a diferencia del SNC, el PIRS no brinda garantías para el desarrollo de RIG cooperativas ni la promoción de la descentralización ni de la participación comunitaria. De hecho, el propio gobierno nacional impulsó que este nivel podía asumir la totalidad de los montos de las obras (Ley 698, 2001, art. 7).

Al igual que el anterior sistema, el PISR fue objeto de demandas de inconstitucionalidad. El actor de dicha demanda, Álvaro Uribe Vélez, argumentó que el PISR violaba diversos artículos sobre los principios del régimen presupuestario, la democracia e instrumentos internacionales. Al igual que en la sentencia de constitucionalidad sobre el SNC (Sentencia C - 562-1998), el magistrado ponente y la mayoría de la Corte se apegaron a la estricta literalidad y desestimó la demanda sin entrar a estudiar a profundidad el sistema y los posibles efectos negativos (Sentencia C 1168 de 2001). No obstante, en esta oportunidad algunos magistrados salvaron el voto al reconocer la vulneración

FINDETER, Banco Agrario, Instituto Nacional de Vivienda, entre otros.

11 Durán y Montenegro (2002) señalan como entidades ejecutoras a aquellas organizaciones que recibieron las partidas en el presupuesto nacional, no necesariamente quienes se encargarán de los temas contractuales. 
de diversas normas y principios constitucionales del presupuesto y advirtieron de los efectos negativos del PIRS.

Ahora bien, al intentar realizar cálculos sobre este sistema de PD y tratar de estimar la influencia de los congresistas en los mismos es una tarea imposible, toda vez que no se cuenta con transparencia ni con los datos mínimos para emprender un análisis, tanto en los primeros años del sistema, como igualmente en la actualidad. Y es que, sin duda, este tercer sistema de política distributiva, es de lejos el menos transparente de los que se han revisado.

A pesar de lo anterior, se mostrarán algunas cifras sobre el PIRS. Para el año de su introducción 2001, los 300 mil millones de pesos equivalían a un $\mathbf{0 . 0 0 5 \%}$ del total de 62 billones de pesos del presupuesto. El valor manejado por congresista sería de 1,132,075,472 pesos o 492,207 dólares. Lo que paradójicamente es una cifra semejante al promedio actual de los CDF a nivel mundial (véase anteriormente el gráfico 2).

Por su parte, si se tomara el total del PISR del año 2001 y no sólo de los 300 mil millones adicionales solicitados por los congresistas como modificación al presupuesto, se estaría hablando de una cifra de casi 10 billones de pesos, lo que equivale al $15 \%$ del total del presupuesto. No obstante, realizar este tipo de cálculos no refleja la realidad, ya que no todas las partidas globales del PISR están dirigidas a inversión de obras de infraestructura ni tampoco transfiere los recursos a los entes territoriales para que éstos se encarguen de la contratación.

Lamentablemente, esto es un elemento poco destacado por diversos actores políticos al proponer reformas a las PD en Colombia. En los siguientes párrafos se realizará dos ejercicios estimativos sobre estos puntos frente a los datos aportados por actores políticos, para demostrar que quizá en el debate público se esté sobredimensionando el papel de los legisladores en el manejo de los recursos del PIRS susceptibles de clientelismo y corrupción.

Por ejemplo, entre los documentos de los promotores de la Consulta Popular Anticorrupción 2018 se puede encontrar la siguiente gráfica sobre la "mermelada": 
Gráfica 3. Esquema sobre bolsas de financiamiento público en Colombia según Comité de Promotores de Consulta Popular Anti Corrupción.

\section{Dl gasto del gobierno nacional se ejecuta mediante 3 bolsas}

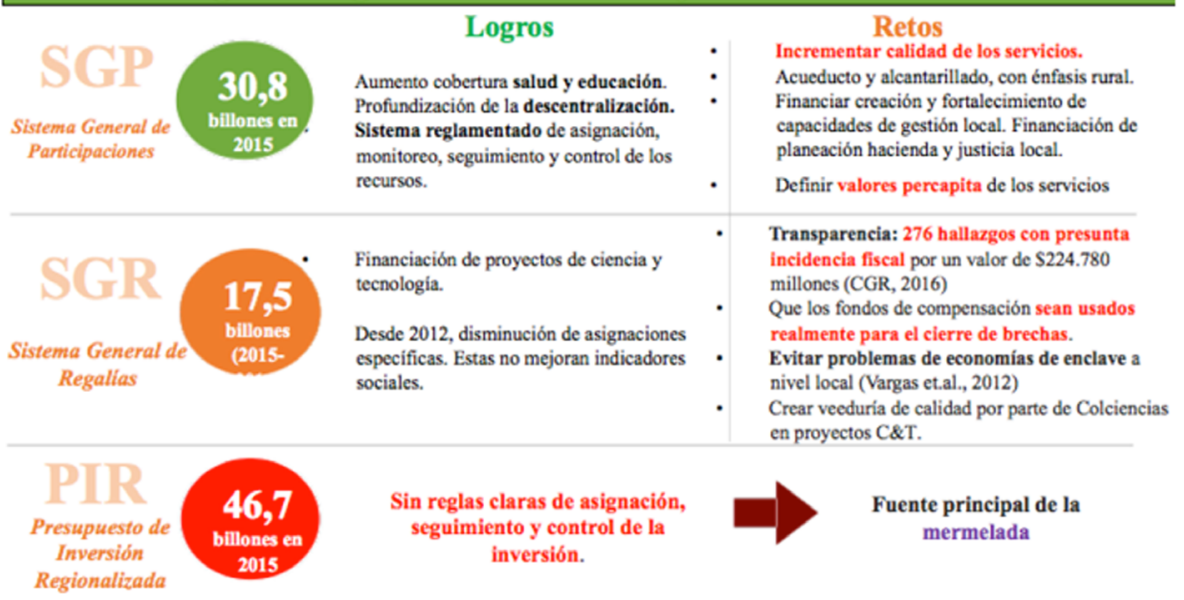

Fuente: Consulta Anticorrupción, 2018, p. 16. En línea: https://bit.ly/2NhKlvV . (Consultado el 10 de enero de 2018)

Como se observa, los promotores de la consultan enfatizan el monto y las características poco transparentes del funcionamiento del PISR, al señalar que es la principal fuente de mermelada. No obstante, de esta bolsa principal de $46,7^{12}$ billones de pesos vale la pena preguntarse ¿Cuánto es "mermelada" o proyectos de inversión gestionados por los congresistas a través de cofinanciación?

Para responder estas preguntas se realizó una selección, preliminar y sin el nivel de minuciosidad deseado ${ }^{13}$, de las partidas para proyectos de inversión en el PISR que posiblemente serían gestionadas y ejecutadas mediante cofinanciación para el año 2017. Los criterios para tomar estas partidas globales de gasto fueron: la relación con la promoción de obras de infraestructura física y la identificación del uso de la cofinanciación al revisar las normas que regulan las partidas.

12 Los promotores de la consulta anticorrupción tomaron el valor correspondiente al PISR de 2014. El valor para el 2015 fue de 49.2 billones (DNP, 2015 disponible en: https://bit.ly/34zvhzE )

13 Como se ha señalado en oportunidades anteriores, en Colombia el nivel de desagregación del presupuesto es mínimo y se limita a partidas globales lo cual dificulta un análisis pormenorizado de los proyectos de inversión. 
Gráfica 4. Posibles Partidas de Inversión Social relacionadas con proyectos de inversión y cofinanciación año 2017. Posiblemente puedan ser objeto de mermelada

\begin{tabular}{|l|l|l|}
\hline $\begin{array}{c}\text { Sector / Ministerio } \\
\text { / Departamento } \\
\text { Administrativo }\end{array}$ & \multicolumn{1}{|c|}{ Partida } & $\begin{array}{c}\text { Montos en pesos } \\
\text { / Nacional PIRS } \\
\mathbf{2 0 1 7}\end{array}$ \\
\hline Educación & Infraestructura Educativa & $391,000,000,000$ \\
\hline Hacienda & Fondo de Adaptación & $1,081,414,839,000$ \\
\hline $\begin{array}{l}\text { Inclusión y reconcil- } \\
\text { iación }\end{array}$ & $\begin{array}{l}\text { Implementación de Obras para la Pros- } \\
\text { peridad }\end{array}$ & $580,529,000,000$ \\
\hline Transporte & Todas las partidas. & $453,622,549,726$ \\
\hline $\begin{array}{l}\text { Vivienda, ciudad y } \\
\text { territorio }\end{array}$ & $\begin{array}{l}\text { Asistencia técnica y apoyo financiero a } \\
\text { proyectos específicos de acueducto y } \\
\text { alcantarillado }\end{array}$ & $310,094,958,238$ \\
\hline & $\begin{array}{l}\text { Total. Posibles partidas de proyectos } \\
\text { de inversión y cofinanciamiento. }\end{array}$ & $2,816,661,346,964$ \\
\hline & $\begin{array}{l}\text { Porcentaje posibles partidas de inver- } \\
\text { sión / Total de PISR. }\end{array}$ & $\mathbf{8} \%$ \\
\hline & $\begin{array}{l}\text { Total Nacional de Presupuesto de } \\
\text { Inversión Social Regional. }\end{array}$ & $35,200,466,785,566$ \\
\hline
\end{tabular}

Fuente: Elaboración propia con base en DNP-PISR 2017.

Estas partidas, que posiblemente sean objeto de caer en el mecanismo de la mermelada, representan el 8\% del total del PISR. La misma continúa siendo elevada en comparación con el monto del DNP sobre las fuentes de ingreso de los entes territoriales, donde la cofinanciación alcanza apenas un 3\% entre los años 2013, 2014 y 2015 (Levitas, 2017).

Otro ejemplo de posible sobre estimación fue el informe denominado Carrusel de la Reelección Resumen por Representante (2014) socializado por el Ex Presidente Álvaro Uribe en 2014 y usado no sólo por el político; sino por analistas como Valencia y Ávila (2014). En este informe se señalaba, entre otros temas, los montos entregados en proyectos de inversión en obras y vías.

Por sólo tomar un caso del informe anterior, a un Senador de la costa caribe colombiana ${ }^{14}$ le es atribuido el manejo de recursos por un valor de $47,525,000,000$ pesos. Si se realizara el mismo ejercicio de estimar las partidas

14 Se prefiere omitir nombre del congresistas. 
de inversión regional relacionadas con obras y cofinanciación asignadas para el departamento en el cual este Senador concentra sus votos, estaríamos hablando que el Congresista manejaría 31 \% del total de partidas de inversión susceptibles de mermelada, lo que posiblemente es una sobrestimación.

A pesar de las estimaciones anteriores, analistas políticos como Valencia y Ávila (2014) y el equipo de la Silla Vacía (2018) han establecido que el uso de los cupos indicativos o mermelada es una de las principales herramientas de campaña política utilizada por candidatos al Congreso, toda vez que les permite mantener en relaciones de subordinación a las autoridades locales; usar las obras como publicidad política de sus "buenas gestiones” y financiar sus campañas de manera irregular o ilegal al obtener porcentajes de contratistas.

De la dinámica anterior no han estado exentas las propias entidades nacionales reconocidas como órganos altamente técnicos encargadas de financiamiento. Por ejemplo, FONADE ha estado envuelto en escándalo de mafias y redes de corrupción para la selección de proyectos a financiar (Semana, 2018).

Lo anterior es muestra que sí existe la relación de intercambio informales entre ejecutivo y congresistas; sin embargo, hace falta mayor investigación sistematizada para dar cuenta de los niveles y los efectos reales de dichas relaciones, no sólo en términos de resultados electorales y funcionamiento de la democracia e institucionalidad; sino también en eficiencia del gasto y de las RIG.

\section{Conclusiones}

La revisión de los tres sistemas de PD con queha contado Colombia desde el siglo XX hasta la actualidad muestran el paso de sistemas con algún grado de formalidad y transparencia como los AP a un intento de políticas formales y que promovieran RIG armónicas como el SNC, que lamentablemente no incluyó a los congresistas como un actor más del sistema y, finalmente, la opción menos transparente y más informal de todas como lo es el PISR.

El análisis permitió también comparar la organización administrativa y el funcionamiento formal e informal de estas experiencias con otras PD del mundo que se caracterizan por la integración de los Congresistas como un actor clave y formal del sistema. Se identificó que las relaciones entre el ejecutivo y el 
legislativo siempre existirán y el tema de debate es hacer dicha participación más o menos transparente. Igualmente, se ha llegado a la conclusión que los sistemas de PD colombianos, tercamente y a diferencia de los sistemas en los otros países estudiados, prefieren ocultar la participación de los congresistas y niegan, obstruyen o dificultan la participación comunitaria.

También se identificó una tendencia en la Corte Constitucional a estudiar las demandas de constitucionalidad contra los sistemas de PD con poca profundidad, sin entrar a analizar los argumentos centrales de los actores ni los efectos lógicos. Esto es diferente a otras cortes donde, bien sea declarando exequible (India) o inexequible (Filipinas) la participación formal de los congresistas y los principales argumentos de los actores son estudiados.

Finalmente, el intento de comparar los montos y valores manejados por los sistemas de PD colombiano con otros casos del mundo permitió identificar que los valores manejados en Colombia por los congresistas, en caso que su participación esté presente en todas las asignaciones presupuestales y proyectos sería muy superior al del resto de los países estudiados. Sin embargo, como se advirtió, realizar este tipo de cálculos no corresponde a la realidad y posiblemente se esté sobrestimando el poder de influencia de los congresistas en la relación ejecutivo- legislativo. De hecho, lo más probable es que los últimos dos sistemas de PD en Colombia, al no reconocer a los congresistas como un actor clave del sistema, los coloque al menos en el plano de lo formal en una condición de minusvalía frente al ejecutivo nacional y en el cumplimiento de su función representativa. Lo anterior no desconoce que desde las relaciones informales ciertos congresistas cuenten con un importante poder de chantaje frente al poder ejecutivo, sobre todo cuando se trata de presidentes que lograron acceder al cargo principalmente por alianzas con grandes electores clientelistas.

Ahora bien, este pequeño aporte es el inicio de toda una serie de investigaciones que abarque las diversas dimensiones de las PD en Colombia. Por ejemplo, un próximo paso necesario dentro de la misma metodología de investigación cualitativa de estudio de caso y con un enfoque de sociología jurídica será estudiar las propuestas de reforma al sistema del PIRS que han surgido desde diversos actores, en especial la iniciativa de los congresistas, que han ido reconociendo poco a poco la necesidad de formalizar el sistema de PD y su participación en el mismo. De igual forma se debe estudiar a profundidad el experimento piloto de RIG de los Gobiernos del Presidente Álvaro Uribe denominados Consejos Comunitarios, los cuales, entre otras actividades, 
contaban con la participación activa y transparente de diversos actores, desde la comunidad, los Congresistas hasta las autoridades y diversos entes de los niveles de gobierno para la identificación de proyectos de inversión y PD.

Otra línea de investigación consiste en ampliar el análisis de las sentencias de control de constitucionalidad sobre los intentos del Congreso de recuperar su iniciativa de gasto o sobre la participación transparente de los congresistas en el experimento de los Consejos Comunitarios.

Finalmente, desde una metodología cuantitativa descriptiva y explicativa es necesario estudiar el comportamiento y la toma de decisiones de los actores locales en la búsqueda de recursos para los municipios y de los funcionarios nacionales que deciden sobre los mismos.

Esta última metodología requiere de ingentes recursos financieros, humanos y tecnológicos que involucren a diversos actores y sectores. Sin embargo, los aportes en términos de conocimiento aplicado serán ampliamente útiles para la cada vez más necesaria reforma del sistema de PD en Colombia.

\section{Referencias}

Aponte Olivella, M. A. (1983). Los auxilios parlamentarios para el desarrollo regional. Tesis para optar al título de Abogado. Bogotá: Universidad Santo Tomás.

Argullol, E. (2004). Federalismo y Autonomía. Barcelona: Ariel.

Baskin, M. y Mezey, M. (2014). Distributive politics in developing countries. Almost Pork. Maryland: Lexington Books.

Baskin, M. (2014). Introduction: are constituency development funds a policy tool?. En Baskin, M. y Mezey, M. (Eds.), Distributive politics in developing countries. Almost Pork, (pp. 1-26). Maryland: Lexington Books.

Bertelli, A., y Grose, C. (2009). Secretaries of Pork? A New Theory of Distributive Public Policy. The Journal of Politics. 71(3), 926-945.

Blair, H. (2014). Constituency needs, constitutional propierty and clientelist patronage: Constituency Development Funds in India. En Baskin, M. y Mezey, M. (Eds.) Distributive politics in developing countries. Almost Pork, (pp. 167-198). Maryland: Lexington Books.

Bonilla-Mejía, L., y Higuera-Mendieta, I. (2017) Political alignemnt in the Time of Weak Parties: Electoral advantage and subnational transfers in Colombia. En Documentos de trabajo sobre economía regional. Banco de la República, (260).

Cárdenas, M., Mejía, C. y Olivera, M. (2006). La economía política del proceso presupuestal en Colombia. Working papers serie - Documento de trabajo. (31). Bogotá: Fedesarrollo. 
Comité de la Consulta Popular Anticorrupción Vence al Corrupto. (2018). Justificación de la Consulta Anticorrupción. Bogotá: Comité de la Consulta Anticorrupción Vence al Corrupto. Recuperado de: https://bit.ly/2NhKlvV .

Corzo Virviescas, M. (1997). Descentralización y fondos de cofinanciación. Crisis coyuntural. (Tesis de grado para optar al título de Economista, Uniandes, Bogotá, Colombia).

Durán Acosta, V., y Montenegro Ceballos, A. M. (2002). Partidas de Inversión Social Regional. (Trabajo de grado para optar al título de Abogado Pontificia Universidad Javeriana, Bogotá, Colombia).

Evans, D. (2014). Earmark in the United States. En Baskin, M. y Mezey, M. (Eds.) Distributive politics in developing countries. Almost Pork, (pp. 107-142). Maryland: Lexington Books.

Galvis, M., Medina, C., y Pallares, C. (2009). Poder regional y coaliaciones en el Plan Nacional de Inversiones Públicas: 1998-2010. Disponible en: https://bit.ly/2Nn5Qvv

Golden, M., y Min, B. (2013). Distributive politics around the world. The Annual Review of political Science, (16), 73-99.

Gómez Barón, W. M. (2003). La intermediación política para la asignación de recursos públicos en Colombia. Virtud, perversión o reconocimiento de una realidad que puede articularse al desarrollo social. (Tesis para optar al título de Magister en Ciencia Política, Uniandes, Bogotá, Colombia).

Gonzalez, L.y Mamone, M. (2015). Who distributes? Presidents, Congress, Governors, and the politics of distribution in Argentina and Brazil. Revista Ibero-Americana de Estudios Legislativos. (4), 17-32.

González Hernández, C. J. (2001). La cofinanciación en Colombia: Una presa más del clientelismo político. (Tesis de grado para optar al título de Politólogo, Uniandes, Bogotá, Colombia).

Hána, D. (2014). Local development implications of pork-barrel: A case study of Czech District. AUC Geographica, 49 (2),73-86.

Hallerberg, M.; Scartascini, C. y Stein, E. (2010). ¿Quiénes deciden el presupuesto? La economía política del proceso presupuestario en América Latina. Colombia: BID.

International Budget Partnership. (2017). Encuesta Open Budget. Encuesta por país Recuperada de: https://bit.ly/2NHF93s .

La Silla Vacía. (2018). El dulce poder. Así funciona la política en Colombia. Bogotá: Aguilar.

Leal B., F. y Dávila L., A. (1990). Clientelismo El sistema Político y su expresión regional. Bogotá: Tercer Mundo Editores e Instituto de Estudios Políticos y Relaciones Internacionales.

Leguizamo, A. (1997). Los fondos de cofinanciación como herramienta de gasto social en Colombia. (Tesis de grado para optar al título de Economista, Uniandes, Bogotá, Colombia).

Leyva Botero, S. (2011). Hacia un nuevo debate sobre la descentralización en Colombia: el análisis intergubernamental desde una lectura institucional. Revista CS, (8), 211-243.

Leyva Botero, S. (2012). Las relaciones intergubernamentales en Colombia (2002-2010): un análisis de los concejos comunales de gobierno. Revista Análisis Político, (76), 119-138.

Levitas, T. (2017). Towards a more equitable and responsive system of intergovermmental fiscal relations in Colombia. Policy Note, SKL - FOINCIDE. Disponible en: https:// bit.ly/2pVvpLb 
Mejía, L.; Botero, F. y Rodríguez Raga, J.C. (2008). ¿Pavimentando con votos? Apropiación presupuestal para proyectos de infraestructura vial en Colombia, 2002-2006. Colombia internacional, (68), 14-42.

Ndii, D. (2014). Money for small things: Experience and Lessons of Kenya`s Constituency Development Fund. En Baskin, M. y Mezey, M. (Eds.) Distributive politics in developing countries. Almost Pork, (pp. 49-82). Maryland: Lexington Books.

Ortega Cárdenas, A. (2010). Hacienda pública las finanzas del Estado. Bogotá: ECOE ediciones.

Pubiano, N. y López, L. M. (2004). Instituciones políticas y presupuesto público: el caso Colombia, 1990-2003. Ensayos sobre política económica. (46-II), 306-356.

Ramírez Plazas, J. (2015). El presupuesto público en Colombia. Bogotá: Librería ediciones del profesional.

Restrepo, J. C. (2014 a). Derecho presupuestal colombiano. Bogotá: Legis.

Restrepo, J. C. (2014 b). Los cupos indicativos a la luz del derecho presupuestal. Ámbito Jurídico. Recuperado de: https://bit.ly/34zlQA5 .

Sánchez Cubides, P. A. (2015). Hacienda pública territorial. Bogotá: Grupo Editorial Ibañez.

Sciara, G. (2009). Planners and the Pork Barrel: Metropolitan Engagement in and Resistance Congressional Transportation Earmarking. (Tesis de grado para optar al título de Doctor en Planeación de la ciudad y la región, Universidad de Berkeley, California, Estados Unidos).

Semana. (2018). Seis de los procesados por "mermelada tóxica en Fonade aceptaron cargos. Recuperado de: https://bit.ly/32mlev0.

Sin autor. (2014). Informe Carrusel de la Reelección. Resumen por representante. Recuperado de: https://bit.ly/36ALBBV .

Ugalde, V. (2011). Capacidades administrativas de los gobiernos locales en el marco de las relaciones intergubernamentales en Estados iberoamericanos. En Cravacuore, D. y Navarro, C. (eds.). Gobierno locales y relaciones intergubernamentales en Iberoamérica. Las bases formales de los procesos de gobernanza local (pp. 41-67). Buenos Aires: Mino y Dávilas Editores.

Valencia, L. y Ávila, A. (2014). Herederos del mal clanes, mafias y congreso 2014-2018. Bogotá: Ediciones B.

Vargas, J. E. (1999). Las relaciones entre el ejecutivo y el legislativo - Un análisis económico. En: Gandour. M. y Mejía. L. B. (Comp.). Hacia el rediseño del Estado, (pp. 225-250). Bogotá: DNP y Tercer Mundo Editores. 